\title{
Comparative in vitro antioxidant activity of Natural and Cultured Ophiocordyceps sinensis
}

\author{
Seema Singh ${ }^{1,3 *}$, Mohommad Arif ${ }^{2}$, Subir Ranjan ${ }^{1}, \&$ Mohammed Nasim ${ }^{2}$ \\ ${ }^{1}$ Defence Institute of Bio-Energy Research (DIBER), Field Station, Pithoragarh, Uttarakhand, India. \\ ${ }^{2}$ Defence Institute of Bio-Energy Research (DIBER), HQ, Haldwani, Uttarakhand, India. \\ ${ }^{3}$ Deoghar College, Deoghar under Sido Kanhu Murmu University, Jharkhand, India. \\ *Correspondence E-mail : seemasinghania96@gmail.com
}

\begin{abstract}
Ophiocordyceps sinensis is a high valued therapeutic fungus endemic to the alpine ecosystems of the Tibetan Plateau and surrounding Himalayas at an altitude of $3000-5000 \mathrm{msl}$. This fungus is widely used as an excellent performance enhancer in traditional Chinese medicine (TCM). The objective of this work was to determine and compare the antioxidant potential of methanolic extract of natural and in vitro cultured mycelium of $O$. sinensis. The antioxidant capacity of the samples was assessed using 2, 2-Diphenyl-1- pikrylhydrazyl (DPPH) free radical scavenging assay, hydroxyl radical eliminating ability, ferrous ion chelating activity and 2, 2'- azino-bis- [3-ethylbenzthiazoline-6sulphonic acid) (ABTS) free radical scavenging assay. The methanolic extract of in vitro cultured $O$. sinensis showed enhanced antioxidant potential than the natural samples. The $\mathrm{IC}_{50}$ value of natural and in vitro cultured samples were $2.98 \mathrm{mg} / \mathrm{ml}$ and $2.81 \mathrm{mg} / \mathrm{ml}$ against DPPH, $3.28 \mathrm{mg} / \mathrm{ml}$ and 2.95 $\mathrm{mg} / \mathrm{ml}$ against Hydroxyl radical and $4.94 \mathrm{mg} / \mathrm{ml}$ and $4.65 \mathrm{mg} / \mathrm{ml}$ against ABTS free radicals respectively. Both natural and cultured sample extract showed $81 \%$ and $86 \%$ iron chelating activity at $1.6 \mathrm{mg} / \mathrm{ml}$ concentration. The results obtained herein allow one to conclude that in vitro cultured $O$. sinensis can be used as a good source of antioxidants as compared to natural one. Further, the better antioxidant activity suggests its role in preventing disorders like cancer, necrosis and aging, induced by reactive oxygen species (ROS).
\end{abstract}

Keywords: $O$. sinensis, in vitro, antioxidant activity, ROS, methanolic extract, free radical.

\section{Introduction}

Ophiocordyceps sinensis (Sung et al., 2007) is one of the most famous traditional Chinese medicines (TCM) and health foods. The fungus parasitizes larvae of moths (Lepidoptera), especially Thitarodes $s p$ (Hepialus armoricanus), and converts each larva into a sclerotium, from which the stroma and finally fruiting body of fungus grows. The complex of larva and fungus has been used as a traditional medicine in China for hundreds of years. The fungus is endemic to the alpine habitats of the Tibetan Plateau above $3000 \mathrm{~m}$ in south-western China, Nepal and India. In India especially it is found in Uttarakhand, Sikkim and Himachal Pradesh (Winkler, 2009). In India this fungus came to the lime light almost one decade earlier when it was collected from the high altitude hills of Dharchula (Uttarakhand) in the Central Himalayas by some local people called Khampas (a Tibetan race). Till date many more places along the Indo-Tibet and IndoNepal boarder namely Laspa, Darti, Milam, Burfu, Mapa, Tola, Ralam, in Johor valley and 
Nagindhura, Galfa, Bona, Chhipalakedar in the basement of Panchachuli in Munsyari and Brahmakot, Najiri, Chhipalakot in Darma valley of Dharchula were also identified as the places of its occurrence (Negi, 2009).

The medicinal properties of $O$. sinensis have been the subject of global research since the 1990s. It is now the world's most costly medicinal mushroom and is protected as an endangered species in China. In recent years it has been regarded as the Himalayan Viagra, which has caused the price to reach USD $\$ 6.77$ per piece of wild medicine. However, the annual harvest of $O$. sinensis has been steadily declining because of its highly specific growth environment, restricted geographical distribution and increasing public demand. The conservation and sustainable harvest are important issues (Panda and Swain, 2011). There is need for research on biological screening; a better understanding of the status in natural habitats, and artificial cultivation of the fungus. So In vitro culture of the fungus has been employed increasingly which possess the same functions as wild herbs (Yang et al., 2005).

Over 20 bioactive ingredients found in O.sinensis which includes ergosterol (Panda and Swain., 2011), extracellular polysaccharides (Zhong et al., 2009), intracellular polysaccharides (Dong and Yao., 2011), cordycepin (Zhou et al., 2009), adenosine (Zhang et al., 2010), guanosine (Zhang et al., 2012), cordymin (Shrestha et al., 2010), lovastatin (Jin et al., 2005), Yaminobutyric acid (Xu and Li., 2010), sitosterol (Chen et al., 2010), myriocin (Liu et al., 2007), melanin (Xu et al., 2006), and serine protease (Shrestha et al., 2012).

Over 30 different bioactivities have been reported for $O$. sinensis, including antidiabetics (Panda and Swain., 2011), immunomodulatory (Yang et al., 2005), immunosuppressive (Dong and Yao., 2011), anticomplementary (Zhou et al., 2009), antitumor (Zhang et al., 2010), antiinflammatory (Zhang et al., 2012), antioxidant (Shrestha et al., 2010), antibacterial (Jin et al., 2005), hepatoprotection ( $\mathrm{Xu}$ and Li., 2010), kidney benefitting (Chen et al., 2010), hypocholesterolemia (Cannon et al., 2009), antiarteriosclerosis (Au et al., 2012), antithrombus (Sharma, 2004), hypotension and vasorelaxant (Winkler, 2008), lung benefitting (Winkler, 2010), antifatigue (Canney, 2006), antiasthma (Wang and Shiao., 2000), erythropoiesis (Zhu et al., 1998), antiarrhythmia (Shrestha et al., 2012), antiaging (Das et al., 2010) and testosterone production (Wang and Liu., 2009). Some reports have indicated that polysaccharides of this fungus exhibiting anti-oxidant activity and nucleosides that inhibit platelet aggregation (Wu et al., 2005).

Li et al., (2001) reported that C. sinensis mycelia manifest antioxidant activity in the xanthine oxidase, haemolysis and lipid peroxidation assay systems. Subsequently, $\mathrm{Li}$ et al., (2002) showed that the fruiting body part and the caterpillar part of $C$. sinensis are similar in chemical composition and antioxidant activity because the mycelia have invaded the caterpillar. Cho et al., (2003) reported that Cordyceps extract possesses 2, 2- diphenyl-1-picrylhydrazyl radical scavenging activity. Wang et al (2005) also noted that the ethanol extract of $C$. sinensis exhibits free radical scavenging activity. Fraction $\mathrm{R}$ derived from the ethanolic extract, at a concentration of $2 \mathrm{mg} / \mathrm{mL}$, scavenged $93 \%$ of 2, 2-diphenyl1-picrylhydrazyl free radicals. Cordyceps species contains a protein-bound polysaccharide that inhibits lipid peroxidation and increases the activity of antioxidant enzymes, such as catalase and superoxide dismutase, in the liver (Shin et al., 2001).

Oxidative damage attributed to reactive oxygen species (ROS), has been broadly recognized as a contributor to cellular necrosis and a variety of pathological conditions such as cancer, degenerative disease in neurons, hepatopathies, atherosclerosis and even aging (Pryor, 1986). This could lead to number of investigations in the study of protective actions on free radical and ROS. Antioxidant plays an important role in preventing the diseases induced by the ROS (Willcox et al., 2004), which result to oxidative damage to DNA, protein and other macromolecules. There are serious concerns about the carcinogenic potential of synthetic antioxidants which is widely used in the food industry, like, butylated hydroxyanisole (BHA), butylated 
hydroxytoluene (BHT). Therefore the natural source of antioxidant is required in place of synthetic one. Rigorous research has been carried out to build up natural alternatives, to fight carcinogenesis and aging processes (Thompson and Moldeus, 1998; Witschi, 1996).

Earlier research work also revealed that OS is good source of antioxidant (Yamaguchi et al., 2000; Li et al., 2001). Previous report highlighted the protective effect between cultured and natural OS samples against oxidative damages on biological macromolecule (Hui et al., 2006). Some reports are also available for antioxidant activity, from aqueous extract of cultivated fruit bodies of Cordyceps militaris (Zhan et al., 2006). However the reports on the mechanism underlying the ROS scavenging potential of methanolic extract of natural and cultured OS samples are not available. Due to increasing concern in using natural antioxidant to scavenge ROS, there is a need to obtain an overall assessment of antioxidant activity of the methanolic extract from natural and cultured (in vitro) mycelium powder of $O$. sinensis.

The present study is aimed to investigate and compare, the in vitro antioxidant potential of methanolic extract of natural and cultured $O$. sinensis using 2, 2-Diphenyl-1- pikryldrazyl (DPPH) free radical scavenging assay, hydroxyl radical eliminating capability, ferrous ion chelating activity and 2, 2'- azino-bis- [3ethylbenzthiazoline-6- sulphonic acid (ABTS) free radical scavenging assay.

\section{Materials and Methods}

\section{Chemicals}

DPPH, ABTS, Quercetin and 2-deoxyribose were purchased from Sigma-Aldrich (Steinheim, Germany); BHT, EDTA (Purity > 99.0\%), and ascorbic acid (Chemical grade); from Loba Chemicals (Mumbai India); hydrogen peroxide $\left(\mathrm{H}_{2} \mathrm{O}_{2}\right)$ (Analytical grade); potassium per sulfate; ferric chloride; ferrous chloride; Ferrozine; TCA (Analytical grade); TBA (Purity > 98.0\%) from, SRL (Mumbai India) were used in the present investigation.

\section{Preparation of the extract}

Natural samples of $O$. sinensis were collected from Laspa region (N-30 $17^{\prime} 06.59^{\circ}$ and E-80 $11^{\prime} 27.2^{\circ}$ ) of Pithoragarh district in Uttarakhand state of India. These samples were washed well with sterile double distilled water, lyophilized for $24 \mathrm{~h}$ and pulverized into powder form i.e. natural mycelial powder.

The in vitro culture of the fungus was established in the laboratory on potato dextrose (PD) medium (pH 5.5 before autoclaving) using stromae tissue as an inoculums. The cultures were incubated at $10^{\circ} \mathrm{C}$ for 30 days and then harvested by centrifugation (20 min; $8000 \mathrm{~g}$ ). The harvested biomass was lyophilized after repeated washing with distilled water and then the dried mycelium was pulverized into fine powder.

One gram of each sample (natural and cultured) was extracted with $100 \mathrm{ml}$ of methanol at room temperature with agitation for $8 \mathrm{~h}$, and the extraction was repeated for three times. The extracts were filtered using Whatman no. 1 filter paper and further concentrated under reduced pressure below $35^{\circ} \mathrm{C}$ and stored in the dark at $4{ }^{\circ} \mathrm{C}$ till use in the experiments. Antioxidant activity of $O$. sinensis was expressed on the basis of sample dry weight.

\section{Scavenging effect on DPPH radicals}

The scavenging effect of the methanolic extract on DPPH radicals was determined using the earlier reported method (Hatano et al., 1989) with some modifications. DPPH solution $(0.1 \mathrm{mM})$ was freshly prepared in methanol. Different concentrations of the extract ranging from $0.05-2.2 \mathrm{mg} / \mathrm{ml}$ were added at an equal volume to methanolic solution of DPPH. The mixture was shaken immediately after adding DPPH and allowed to stand at room temperature in the dark for 30 $\min$. The decrease in absorbance at $517 \mathrm{~nm}$ was then measured using a spectrophotometer (LaboMed Inc, Culvar city, USA). The experiment was repeated three times using Ascorbic acid as control. The absorbance was measured at $517 \mathrm{~nm}$. The percent antioxidant or radical scavenging activity was calculated using the following formula: 


\section{$\underline{A c-A s} \times 100$}

$A C$

Where, Ac and As are the absorbance of control and sample, respectively.

\section{Scavenging effect on hydroxyl radicals}

Scavenging effect on hydroxyl radicals was carried out by using Halliwell et al., 1987. The $1.0 \mathrm{ml}$ reaction mixture containing $0.4 \mathrm{ml}$ of sodium phosphate buffer $(20.00 \mathrm{mM} / \mathrm{L}, \mathrm{pH}$ 7.4), $0.1 \mathrm{ml}$ of extract (at concentrations of $0.2-2.2 \mathrm{mg} / \mathrm{ml}), 0.1 \mathrm{ml}$ of 2-deoxyribose (60.00 $\mathrm{mM}), 0.1 \mathrm{ml}$ of hydrogen peroxide $(10.00 \mathrm{mM})$, $0.1 \mathrm{ml}$ of ferric chloride $(1.00 \mathrm{mM}), 0.1 \mathrm{ml}$ of EDTA $(1.04 \mathrm{mM})$ and $0.1 \mathrm{ml}$ of ascorbic acid (2.00 mM), was incubated at $37^{\circ} \mathrm{C}$ for $1 \mathrm{~h}$. Solutions of ferric chloride and ascorbic acid were made up immediately before use. The reaction was stopped by adding $1.0 \mathrm{ml}$ of TBA $(1 \%)$ and $1.0 \mathrm{ml}$ of TCA $(2.8 \%)$. The mixture was boiled for $15 \mathrm{~min}$, cooled and then measured for the absorbance at $532 \mathrm{~nm}$. BHT was used as a positive control. The radical scavenging activity was calculated using the equation mentioned in the above section on scavenging effect on DPPH radicals, where $\mathrm{Ab}$, As and Asb are the absorbance at $532 \mathrm{~nm}$ of the blank, extract or BHT, and sample blank respectively.

\section{Ferrous ion chelating activity assay}

The chelating activity of the extract on ferrous ion was carried out by Decker and Welch (1990) method. The $1.0 \mathrm{ml}$ extract (0.05-2.4 $\mathrm{mg}$ ) was mixed with $3.7 \mathrm{ml}$ of deionised water and then the mixture was reacted with $0.1 \mathrm{ml}$ of ferrous chloride $(2.00 \mathrm{mM})$ and $0.2 \mathrm{ml}$ of Ferrozine $(5.00 \mathrm{mM})$ for $20 \mathrm{~min}$. The absorbance at $562 \mathrm{~nm}$ was determined. EDTA was used as positive control and chelating activity on ferrous ion was calculated as the following equation with $A b$ as the absorbance of the blank without extract or EDTA and As as the absorbance in the presence of the extract or EDTA.

Inhibition $(\%)=\underline{A b-A s} \times 100$

$A b$

\section{Scavenging effect on ABTS radicals}

Free radical scavenging capacity of $O$. sinensis was assessed with the ABTS assay (Mohsin et al., 2011). Solution of ABTS in methanol $(0.7 \mathrm{mM})$ and $2.45 \mathrm{mM}$ potassium per sulfate in water were prepared as separate stock solution. The working solution then obtained by mixing two solution in equal amount and allowing them to react for $12 \mathrm{~h}$ at room temperature in the dark. The solution was then diluted by mixing $1.0 \mathrm{ml}$ of $\mathrm{ABTS}^{+}$ solution and an appropriate volume of methanol. The extract $(0.05-2.8 \mathrm{mg} / \mathrm{ml})$ of 1.0 $\mathrm{ml}$ was mixed with $1.0 \mathrm{ml}$ of ABTS solution. The absorbance at $734 \mathrm{~nm}$ was then measured after a 10 minute reaction time. The ABTS scavenging capacity for the extract was compared with Quercetin, as:

$\%$ scavenged ABTS radical $=[A b S$ control Abs sample $/$ Abs control] $\times 100$

\section{Statistical Analysis}

The treatments and controls of the experiments were replicated thrice. Crop Stat for Windows, developed by the Biometrics Unit, IRRI Philippines was used for analysis of variance (ANOVA) for the experiments laid out in Completely Randomized Design (CRD). The treatment means were compared by Least Significant Difference (LSD) Test at a significance level of $P \leq 0.05$.

\section{Results}

\section{Scavenging effect on DPPH radicals}

DPPH radical scavenging activity of the extract was evident at all of the tested concentrations (Figure 1). The scavenging effect increased with the increasing concentrations from 0.05$2.2 \mathrm{mg} / \mathrm{ml}$. The scavenging activities of methanolic extract of natural and cultured mycelium at concentration of $1.4 \mathrm{mg} / \mathrm{ml}$ were found to be near $90 \%$. The methanolic extract of cultured $O$. sinensis was found to exhibit significantly higher scavenging activity than natural extract at this concentration. After the $1.6 \mathrm{mg} / \mathrm{ml}$ concentration both the extracts showed almost similar scavenging activity. The $\mathrm{IC}_{50}$ value of methanolic extract of natural and in vitro cultured sample was $2.98 \mathrm{mg} / \mathrm{ml}$ and $2.81 \mathrm{mg} / \mathrm{ml}$. 


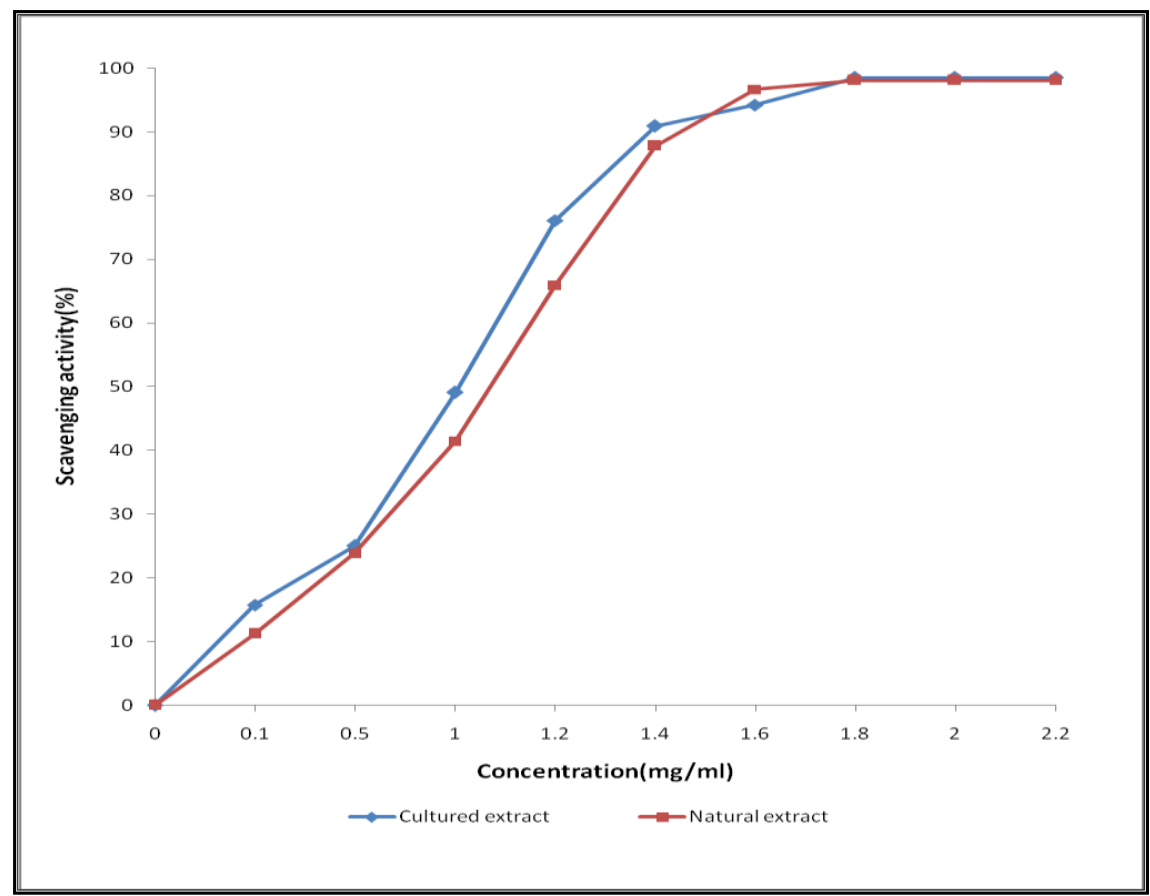

Figure1. The DPPH radical scavenging activity of methanolic extract of natural and cultured O.sinensis.

\section{Scavenging effect on hydroxyl radicals}

As shown in Figure 2, the extract from $O$. sinensis showed hydroxyl radicals eliminating activity in a dose-dependent manner. The scavenging effect increased with the increasing concentrations from $0.05-2.2$ $\mathrm{mg} / \mathrm{ml}$. The scavenging activity of cultured and natural extracts at a concentration of 1.4 $\mathrm{mg} / \mathrm{ml}$ was observed $86 \%$ and $84 \%$ respectively. The scavenging activity of cultured extract was significantly higher than that of natural. The $\mathrm{IC}_{50}$ value of methanolic extract of natural and in vitro cultured sample was $3.28 \mathrm{mg} / \mathrm{ml}$ and $2.95 \mathrm{mg} / \mathrm{ml}$.

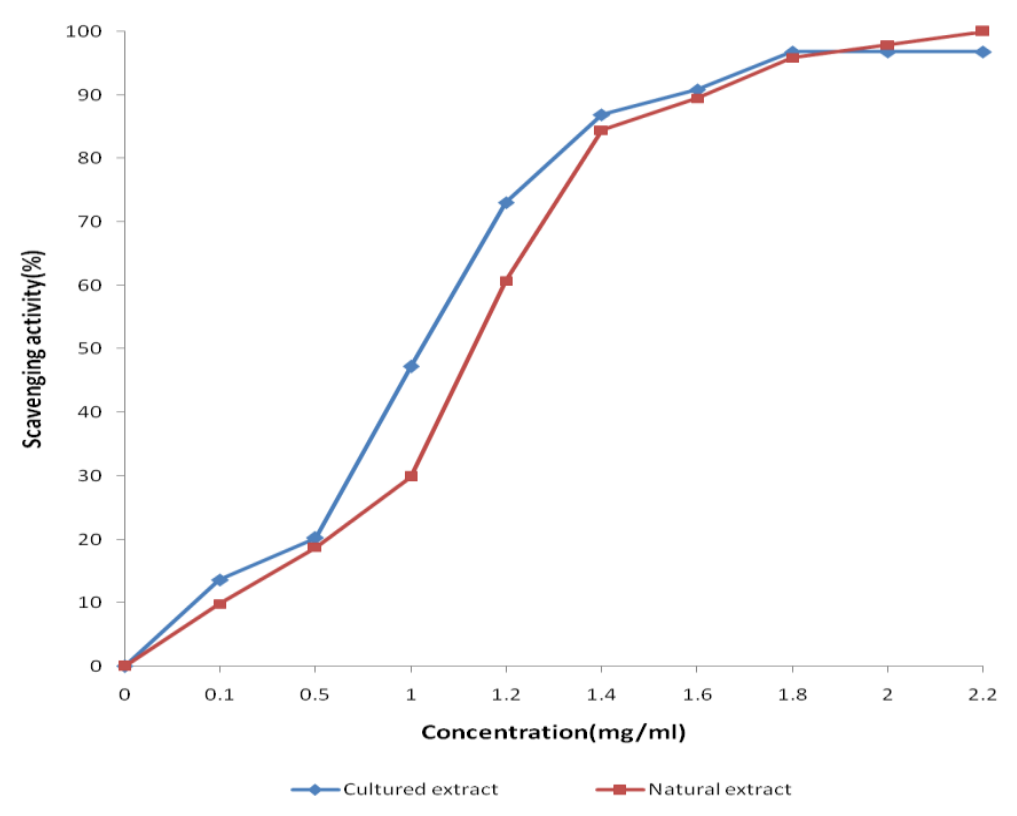

Figure2. The hydroxyl ion eliminating capability of methanolic extract of natural and cultured O.sinensis. 


\section{$\mathrm{Fe}^{2+}$ chelating activity}

As shown in Figure 3, the extract from $O$. sinensis showed chelating activity in a dosedependent manner. The chelating effect increased with the increasing concentrations from $0.05-2.4 \mathrm{mg} / \mathrm{ml}$. The chelating activity of cultured extract was approx $86 \%$ at a concentration of $1.6 \mathrm{mg} / \mathrm{ml}$, significantly higher as compared to of natural extract (approx $81 \%$ ) at the same concentration.

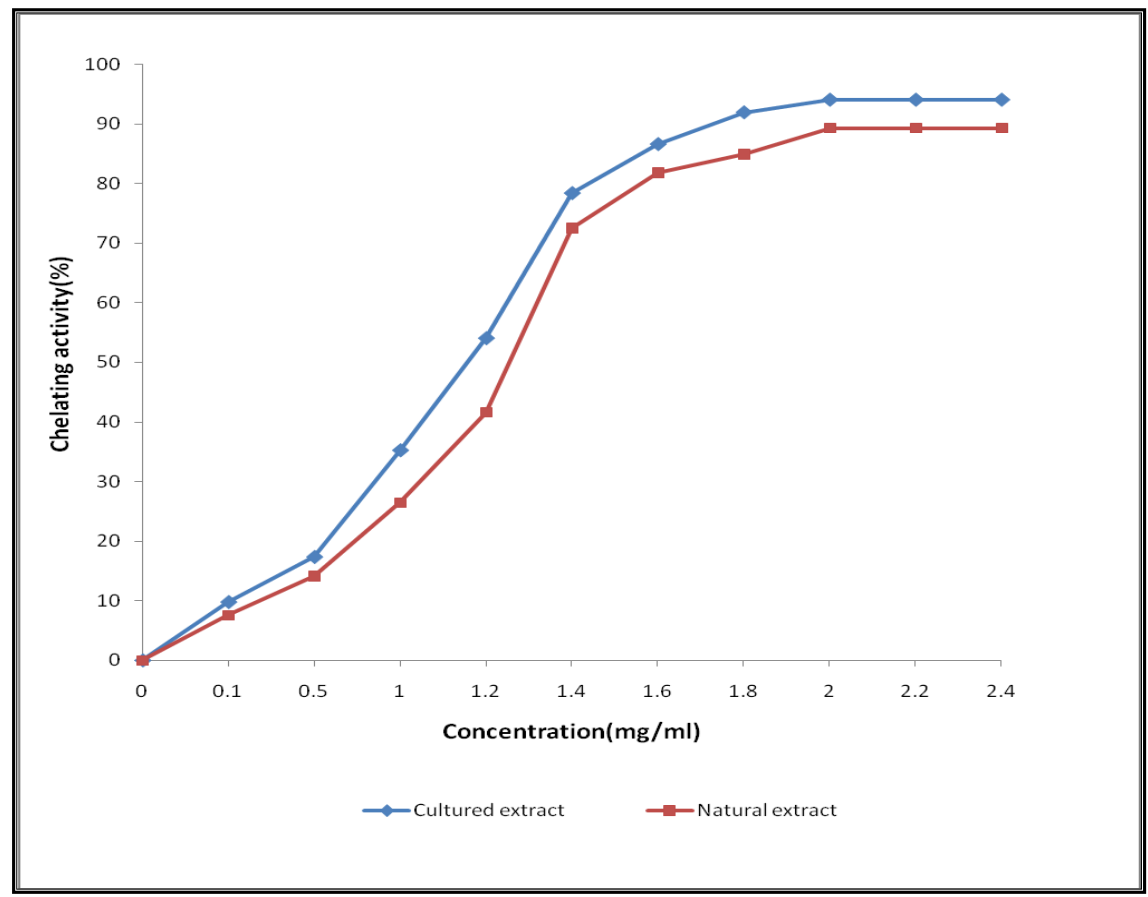

Figure3. The $\mathrm{Fe}^{2+}$ chelating activity of the methanolic extract of natural and cultured $\mathrm{O}$. sinensis.

\section{Scavenging effect on ABTS radical}

Methanolic extract of both natural and cultured $O$. sinensis were evaluated comparatively for their scavenging capacities towards ABTS radical system (Figure.4). All the extracts showed considerable ABTS scavenging capacity in dose dependent manner. The scavenging effect increased with the increasing concentrations from $0.05-2.8$ $\mathrm{mg} / \mathrm{ml}$. The scavenging activities of methanolic extract of cultured and natural mycelium at a concentration of $2.0 \mathrm{mg} / \mathrm{ml}$ were found to be approx $82 \%$ and $81 \%$, respectively. Extract of cultured sample showed significantly higher ABTS radical scavenging than natural samples. The $\mathrm{IC}_{50}$ value of methanolic extract of natural and in vitro cultured sample was $4.94 \mathrm{mg} / \mathrm{ml}$ and $4.65 \mathrm{mg} / \mathrm{ml}$. 


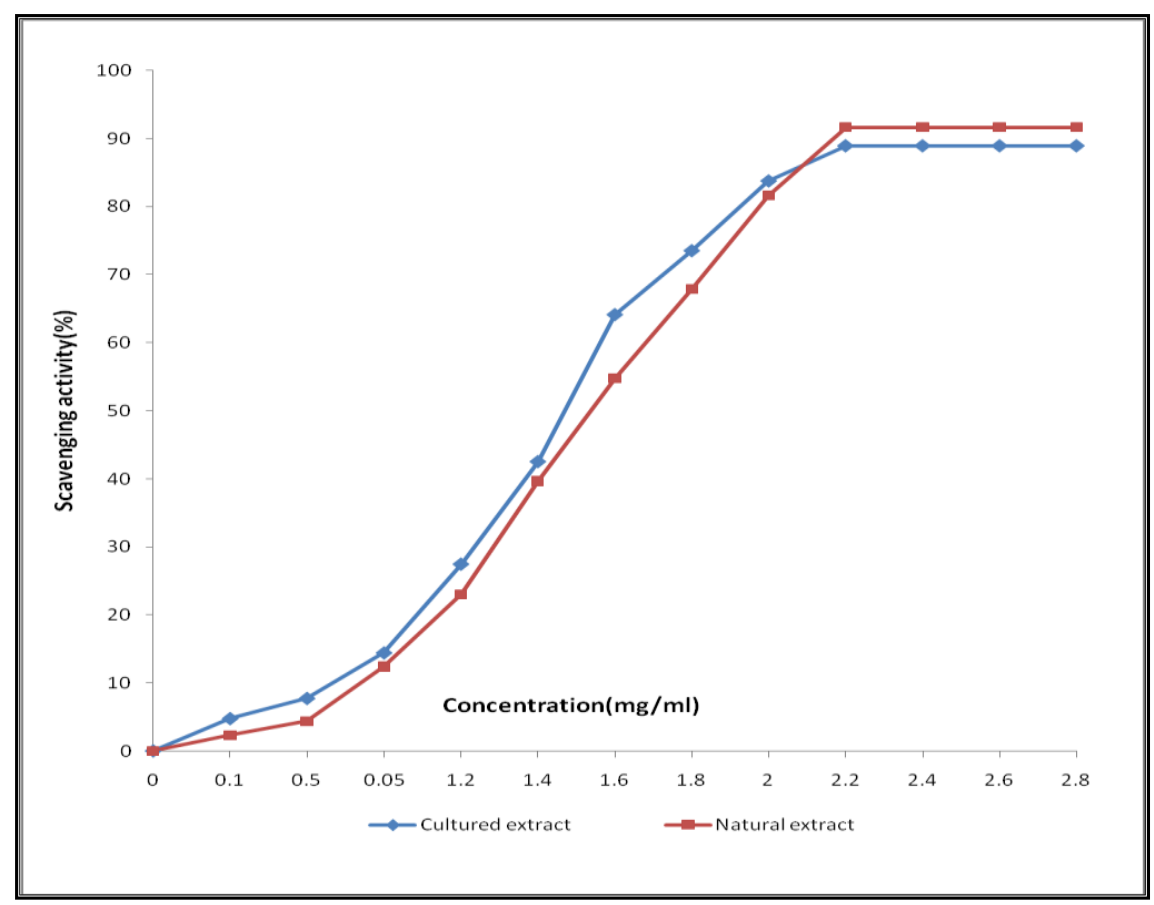

Figure 4. The ABTS scavenging activity of the methanolic extract of natural and cultured $O$. sinensis.

\section{Discussion}

There are frequent methods and modifications available for estimation of antioxidant activity of a compound (Zhan et al., 2006). A single method could not provide a broad picture of the antioxidant profile of a studied sample. The present study was focused to evaluate the antioxidant potential of methanolic extract of natural and cultured O.sinensis using different in vitro radical systems and the results clearly revealed straight and effective antioxidant activity in these samples.

One of the quick methods to evaluate antioxidant activity is the scavenging activity evaluation through DPPH, a stable free radical and widely used index (Mokbel and Hashinaga, 2006). Earlier report revealed that DPPH scavenging activities of aqueous extracts of both natural and cultured mycelium of $C$. sinensis were found to be over $80 \%$ inhibitions at $4-8 \mathrm{mg} / \mathrm{ml}$. Some reports also available for scavenging activity (over $80 \%$ ) of C. militaris against DPPH (Zhan et al., 2006).

The present study showed that the methanolic extract of both natural and cultured $O$. sinensis had a stronger DPPH scavenging ability at the concentration $1.4 \mathrm{mg} / \mathrm{ml}$ but cultured extract had significantly higher scavenging activity than the natural extract of same concentration .

Earlier study had already been done for the antioxidant activities of hot-water extracts from natural and cultured mycelia of $C$. sinensis using six in vitro assays, including inhibition of linoleic acid peroxidation, scavenging abilities on DPPH, hydroxyl and superoxide anion radicals, the reducing power and the chelating ability on ferrous ions. Among these assays, the extracts showed the best effect on the inhibition of linoleic peroxidation with the lowest $\mathrm{IC}_{50}$ values and with an inhibition rate over $90 \%$ at concentration of $0.8-1.6 \mathrm{mg} / \mathrm{ml}$, more stable than that of $\alpha$-tocopherol, a recognised natural antioxidant (Dong and Yao, 2008). The hot water extract of cultivated fruit bodies of $C$. militaris was also reported to be effective on the hydroxyl radicals scavenging (Shen and Shen, 2001) but the effect of scavenging activity of methanolic extract had yet not been clearly known. The methanolic extract of cultured $O$. sinensis had high potential of free-radical scavenging activity than the natural extract. This work clearly demonstrated that a low concentration of cultured $O$. sinensis was found to be effective for free radical scavenging. 
Various studies have shown that polysaccharide is one of the active component in O.sinensis associated with antioxidant activity (Shin et al., 2001; Liu and Shen, 2003). $O$. sinensis is a rich source of Polyphenols. Phenolic compounds have been proven a major class of phytochemicals, which are accountable for inhibiting the oxidative damage caused by the free radicles. The concentrations of phenolic compounds are higher in methanolic extracts when compared with water extracts. All the types of bioactive Polyphenols were present in $O$. sinensis including flavonoids, flavonols and proathocyanidins (Mohsin, 2012).

In this study four different standards namely ascorbic acid, BHT, EDTA and Quercetin were used for a comparative study with methanolic extract of natural and cultured mycelium of $O$. sinensis The natural and cultured methanolic extract showed stronger DPPH scavenging effect than hydroxyl radical, but moderate with ferrous ion chelating and minimum with scavenging activity on ABTS. The $\mathrm{IC}_{50}$ value of the extracts clearly showed that in vitro cultured extract had stronger scavenging activity than that of natural one.

\section{References}

Au, D., Wang, L., Yang, D., Mok, D.K., Chan, A.S., Xu, H. (2012). Application of microscopy in authentication of valuable Chinese medicine I-Cordyceps sinensis, its counterfeits, and related products. Microsc Res Tech, 75. 5464.

Canney. S. (2006). Cordyceps sinensis animal, vegetable or both? J Chin Med, 80.439.

Cannon, P.F., Hywel-Jones, N.L., Maczey, N., Norbu, L., Tshitila, S, T, et al. (2009). Steps towards sustainable harvest of Ophiocordyceps sinensis in Bhutan. Biodivers Conserv, 18. 2263-81.

Chen, J., Lee, S., Cao, Y., Peng, Y., Winkler, D., Yang, D. (2010). Ethnomycological use of medicinal Chinese caterpillar fungus, Ophiocordyceps sinensis (Berk.) G. H. Sung et al. (ascomycetes) in Northern Yunnan Province, SW China. Int J Med Mushrooms, 12. 427-34.
However, in considering the overall antioxidant activity, the methanolic extract of natural and in vitro cultured $O$. sinensis extract can still be a useful bioactive material with competent antioxidant property. The antioxidant activities of the extract in the assays performed in this study prompt the possible uses of the natural and cultured O.sinensis, to meet the needs of the natural antioxidant sources for human health. Natural $O$. sinensis sample are very less in nature. The availability of natural species is decreasing day by day due to uncontrolled utilization from the nature (Singh et al., 2010). Cultured mycelia are easily available due to already established in vitro culture of $O$. sinensis. Therefore cultured mycelia can meet the increasing demand of such a natural antioxidant for human health and nutraceuticals development for pharmaceutical industries. In fact cultured $O$. sinensis is a promising resource for natural antioxidants.

\section{Acknowledgements}

Research fellowship to Seema Singh from DRDO is gratefully acknowledged.

Cho, J., Kang, J.S., Long, P. H., Jing, J., Back, Y., Chung, K.S. (2003)Antioxidant and memory enhancing effects of purple sweet potato anthocyanin and cordyceps mushroom extract. Arch. Pharmacol. Res, 26. 821-825

Das, S.K., Masuda, M., Sakurai, A., Sakakibara, M. (2010). Medicinal uses of the mushroom Cordyceps militaris: Current state and prospects. Fitoterapia. 81. 961-8.

Decker, E.A., Welch, B. (1990). Role of ferritin as a lipid oxidation catalyst in muscle food. $J$ Agr Food Chem, 38. 674-677.

Dong, C.H., Yao, Y.J. (2008). In vitro evaluation of antioxidant activities of aqueous extracts from natural and cultured mycelia of Cordyceps sinensis. Lebenson Wiss Technol, 41. 669-677.

Dong, C.H., Yao, Y.J. (2011). On the reliability of fungal materials used in studies on Ophiocordyceps sinensis. J Ind Microbiol Biotechnol, 38. 1027-35. 
Halliwell, B., Gutteridge, J.M.C., Arugma, O.I. (1987). The deoxyribose method: A simple "Test-Tube" assay for determination of rate constants for reactions of hydroxyl radicals. Anal Biochem, 165. 215-219.

Hatano, T., Edamatsu, R., Mori, A., Fujita, Y., Yasuhara, T., Yoshida, T., Okuda, T. (1989). Effects of interactions of tannins with coexistion substances VI. Effect of tannins andrelated poluphenols on superoxide anion radical, and on 1, 1-diphenylpicryhydrazyl radical. Chem Phar Bull, 37. 2016-21.

Hui, M. Y., Wang, B.S., Huang, S.C., Duh, P.D. (2006). Comparison of Protective Effects between Cultured Cordyceps militaris and atural Cordyceps sinensis against Oxidative Damage. J. Agric. Food Che, 54 (8). 31323138.

Jin, C., Wu, X., Chen, G. (2005). Clinical application of Jinshuibao capsule. Cap Med, 12. 42-3.

Li, S.P., Li, P., Dong, T.T.Z., Tsim, K.W.K. (2001 a) Antioxidation activity of different types of natural Cordyceps sinensis and cultured Cordyceps mycelia. Phytomedicine, 8. 207212.

Li, S.P., Su, Z. R., Dong, T. T., Tsim, K. W. (2002). The fruiting body and its caterpillar host of Cordyceps sinensis show close resemblance in main constituents and antioxidation activity. Phytomedicine, 9. 319-324.

Liu, F., Wu, X.L., Chen, S.J., Yin, D.H., Zeng, W., Zhong, G.Y. (2007). Advances in studies on artificial culture of Cordyceps sinensis. Chin Tradit Herb Drugs, 38. 302-5.

Liu, Y.K., Shen, W. (2003) Inhibitive effect of Cordyceps sinensis on experimental hepatic fibrosis and its possible mechanism. World Journal of Gastroenterology, 9. 529-533.

Mohsin, M. (2012). Biochemical studies of Ophiocordyceps sinensis: An overall performance Enhancing Caterpillar mushroom. DRDO Science Spectrum, 170-175.

Mohsin, M., Negi, P.S., Ahmed, Z. (2011)Determination of antioxdant activity And Polyphenol contents of Wild Lingzhi or Reishi Medicinal Mushroom, Ganoderma Lucidum (W. Curt. Fr.). P.karst. (Higher
Basidiomycetes) from Central Himalayan hills of India. Int Journal of medicinal Mushrooms, 13(6). 535-544.

Mokbel, M.S., Hashinaga, F. (2006). Evaluation of the antioxidant activity of extracts from buntan (Citrus grandis Osbeck) fruit tissues. Food Chem, 94. 529-534.

Negi, P.S. Ethno-mycology Study of Yarsha Gamboo (Cordyceps sinensis (Berk) Sacc.) in Indo-Tibet Border Areas of Uttarakhand. DRDO Technology Spectrum, 161-166.

Panda, A.K., Swain, K. C. (2011). Traditional uses and medicinal potential of Cordyceps sinensis of Sikkim. J Ayurveda Integr Med, 2. 9-13.

Pryor, W. (1986). Oxy-radicals and related species: their formation, lifetimes, and reaction. Annu.Rev. Physiol, 48. 657-667.

Shen, Q.Y., Shen, Q.Y. (2001). Study on the resisting oxygen free radical and hydroxyl free radical effect of Cordyceps militaris. Guihaia, 21. 252-254 (in Chinese with an English abstract).

Shin, K.H., Lim, S.S., Lee, S.H., Lee, Y.S., Cho, S.Y. (2001)Antioxidant and immunostimulating activities of the fruiting bodies of Paecilomyces japonica, a new type of Cordyceps sp. Ann. NY Acad. Sci, 928. 261-273.

Shrestha, B., Zhang, W., Zhang, Y., Liu, X. (2010). What is the Chinese caterpillar fungus Ophiocordyceps sinensis (Ophiocordycipitaceae)? Mycology, 1. 228-36.

Singh, R., Negi, P.S., Ahmed, Z. (2010). Ophiocordyceps sinensis- valuable caterpillar fungus from the Himalayan hills. Correspondance current science, 99(7). 865.

Sung, G.H., Hywel-Jones N.L., Sung, J.M., Luangsaard, J.J., Shrestha, B., Spatafora, J.W. (2007). Phylogenetic classification of Cordyceps and clavicipitaceous fungi. Studies in Mycology, 57. 5-59.

Thompson, D., Moldeus, P. (1988). Cytotoxicity of butylated hydroxyanisole and butylated hydroxytoluene in isolated rat hepatocytes. Biochem Pharmacol, 37. 2201-2207. 
Wang, B.J., Won, S.J., Yu, Z.R., Su, C.L. (2005)Free radical scavenging and apoptotic effects of Cordyceps sinensis fractionated by supercritical carbon dioxide. Food Chem Toxicol, 43.543-552.

Wang, Z., Liu, J.L. (2009). Advances in studies on chemical constituents of Cordyceps. Chin Tradit Herb Drugs, 40.1157-60.

Willcox, J.K., Ash, S.L., Catignani, G.L. (2004). Antioxidants and prevention of chronic disease. Crit Rev Food Sci, 44. 275-295.

Winkler, D. (2008). Yartsa Gunbu (Cordyceps sinensis) and the fungal commodification of Tibet's rural economy. Econ Bot, 62. 291-305.

Winkler, D. (2009). Caterpillar fungus (Ophiocordyceps sinensis) production and sustainability on the Tibetan Plateau and in the Himalayas. Asian Med, 5. 291-316.

Witschi, H.P. (1986). Enhanced tumour development by butylated hydroxytoluene (BHT) in the liver, lung and gastro-intestinal tract. Food Chem Toxicol, 24. 1127-1130.

Wu, Y., Sun, C., Pan, Y. (2005). Structural analysis of a neutral (1-->3),(1-->4)-beta-Dglucan from the mycelia of Cordyceps sinensis. J. Nat. Prod, 68.812-814.

Xu, F.L., Huan, W.Y., Wu, T.X., Qiu, X., Zhang, H., Liu, X.H. (2006). Clinical efficacy of Cordyceps sinensis for chronic kidney diseases: A systematic review. Chin J Evid Based Med, 6. 804-8.

Xu, H., Li, S. (2010). Pharmacological effects of Bailing capsule and its application in lung disease research. Zhongguo Zhong Yao Za Zhi, 35. 2777-81.

Yamaguchi, Y., Kagota, S., Nakumura, K., Shinozuka, K., Kunitomo, M. (2000). Antioxidant activity of the extracts from fruiting bodies of cultured Cordyceps sinensis. Phytother .Res, 14. 647-649.
Yang, J., Zhang, W., Shi, P., Chen, J., Han, $X$. Wang, Y. (2005)Effects of exopolysaccharide fraction (EPSF) from a cultivated Cordyceps sinensis fungus on $\mathrm{C}-$ Myc, c- Fos, and VEGF expression in B16 melanoma-bearing mice. Pathol. Res. Pract, 201. 745-750.

Zhan, Y., Dong, C.H., Yao, Y.J. (2006). In vitro evaluation of antioxidant activities of aqueous extracts from natural and cultured mycelia of Cordyceps militaris. $J$ of Integrative Plant Biology, 48 (11). 1365-1370.

Zhang, Y., Li, E., Wang, C., Li, Y., Liu, X. (2012). Ophiocordyceps sinensis, the flagship fungus of China: Terminology, life strategy and ecology. Mycology, 3. 2-10.

Zhang, Y., Zhang, S., Wang, M., Bai, F., Liu, $X$. (2010). High diversity of the fungal community structure in naturally-occurring Ophiocordyceps sinensis. PLOS ONE, 5.1-8.

Zhong, S., Pan, H., Fan, L., Lv, G., Wu, Y., Parmeswaram, B., et al. (2009). Advances in research of polysaccharides in Cordyceps species. Food Technol Biotechnol, 304 -12.

Zhou, X., Gong, Z., Su, Y., Lin, J., Tang, K. (2009). Cordyceps fungi: Natural products, pharmacological functions and developmental products. J Pharm Pharmacol, 61. 279-91.

Zhu, J.S., Halpern, G.M., Jones, K. (1998). The scientific rediscovery of a precious ancient Chinese herbal regimen: Cordyceps sinensis Part II. J Altern Complement Med, 4.429-57.

\section{Abbrevitions:}

OS, Ophiocordyceps sinensis; msl, mean sea level; TCM, traditional Chinese medicine; DPPH, 2, 2-Diphenyl-1- pikrylhydrazyl; HRE, hydroxyl radical eliminating; ICA, iron chelating activity; ABTS, 2, 2'- azino-bis- [3ethylbenzthiazoline-6- sulphonic acid; reducing power; ROS, reactive oxygen species. 